\title{
Archéopages
}

Archéopages Archéologie et société

$35 \mid 10 / 2012$

Vivre avec les bêtes

\section{Espace rural de la fin de l'âge du Fer}

Jean-Philippe Baigl

\section{(2) OpenEdition}

Journals

Édition électronique

URL : https://journals.openedition.org/archeopages/326

DOI : 10.4000/archeopages.326

ISSN : 2269-9872

\section{Éditeur}

INRAP - Institut national de recherches archéologiques préventives

\section{Édition imprimée}

Date de publication : 1 mai 2013

Pagination : 98-99

ISSN : 1622-8545

\section{Référence électronique}

Jean-Philippe Baigl, «Espace rural de la fin de l'âge du Fer », Archéopages [En ligne], 35 | 10/2012, mis en ligne le 01 octobre 2014, consulté le 22 janvier 2022. URL : http://journals.openedition.org/ archeopages/326 ; DOI : https://doi.org/10.4000/archeopages.326 
Période

Protohistoire

\section{Espace rural de la fin de l'âge du Fer}

Jean-Philippe Baigl

Inrap, responsable de secteur

Site

Les Grands Champs

Coulon

Deux-Sèvres

Date

Début septembre

mi-novembre 2011

$\infty$

Surface fouillée

1 ha

Équipe

Responsable d'opération Céline Pelletier

Archéozoologie

Anna Baudry-Dautry

Dendrologie

Vincent Bernard

Étude statuaire

Alain Duval

Étude céramologique Guilhem Landreau

Malacologie marine

Caroline Mougne

Instrumentum

Olivier Nillesse la plus dense a été prescrite pour la fouille préventive. Le travail de post-fouille étant en cours, les résultats évoqués ne tiennent donc compte que des observations de terrain et des perspectives de recherches.

Comme sur la plupart des sites ruraux de la fin de l'âge du Fer, les vestiges concernent essentiellement des fossés d'enclos [ill. 1] et des trous de poteaux. On signale également quelques fosses, dont certaines liées à une activité de cuisson, deux sépultures à inhumation creusées dans le comblement de fossés et des ossements humains mêlés à du mobilier domestique (faune, céramique...), un puits carré contenant des pièces de bois (chèvre ou éléments pour le puisage de l'eau?) [ill. 2]... En attendant l'étude précise du mobilier, on peut esquisser une évolution chronologique de l'occupation à partir des différentes phases matérialisées par les enclos Lors de la première phase, entre la seconde moitié du III $^{\mathrm{e}}$ et le début du $\mathrm{II}^{\mathrm{e}}$ siècle avant notre ère, les fossés d'enclos atteignent en général $2 \mathrm{~m}$ de large pour $1 \mathrm{~m}$ de profondeur et montrent trois états successifs sur une faible surface dans l'emprise du décapage. Aucun bâtiment n'a été attribué pour l'instant à cette période. La deuxième phase se situe dans la seconde moitié du II ${ }^{\mathrm{e}}$ siècle avant notre ère. Le fossé de l'enclos principal est de dimensions plus imposantes, avec 4 m d'ouverture et $2 \mathrm{~m}$ de profondeur; il dispose de plus d'un talus interne. Pour cette phase, au moins un bâtiment (habitat principal ?) a été identifié et plusieurs enclos périphériques sont à lui associer. La troisième phase comprend la fin du $\mathrm{II}^{\mathrm{e}}$ et le début du ${ }^{\mathrm{er}}$ siècle avant notre ère : l'enclos principal se monumentalise encore avec le creusement d'un fossé de $7 \mathrm{~m}$ d'ouverture et $3 \mathrm{~m}$ de profondeur, toujours avec un talus interne.

Le bâtiment principal, d'au moins $80 \mathrm{~m}^{2}$ au sol, est érigé à l'emplacement de celui de la phase antérieure. Il est construit sur pannes porteuses d'un probable étage et possède un toit à quatre pans. Il s'apparente davantage à un manoir quà un simple bâtiment d'habitation. Il sera par la suite complètement détruit (brûlé) puis reconstruit plus modestement $\left(40 \mathrm{~m}^{2}\right)$ au même endroit, mais l'enclos et le talus interne restent intacts.

De nombreux autres bâtiments sont édifiés dans l'enceinte, appartenant à l'un des deux états de cet enclos. Des enclos périphériques sont également existants lors de cette phase. Parmi le mobilier, on notera en particulier la présence d'un fragment de statue en calcaire [ill. 3], d'une perle en verre, de nombreuses amphores vinaires, de céramique à vernis noir (campanienne $\mathrm{A}$ et $\mathrm{B}$ ),

d'un massacre de cerf (trophée de chasse), d'un statère en or allié [ill. 4], de fibules... Le site est ensuite abandonné. Les fossés de l'enclos principal sont définitivement comblés par le démantèlement rapide et massif du talus, scellant ainsi les derniers niveaux d'occupation
1. Coupe du fossé de l'enclos principal de la phase la plus récente avec 3 séquences de comblement bien lisibles : la fine couche au centre (dépotoir) est scellée par l'important remblai issu du démantèlement du talus. construction d'un lotissement de plusieurs hectares. Seule la partie

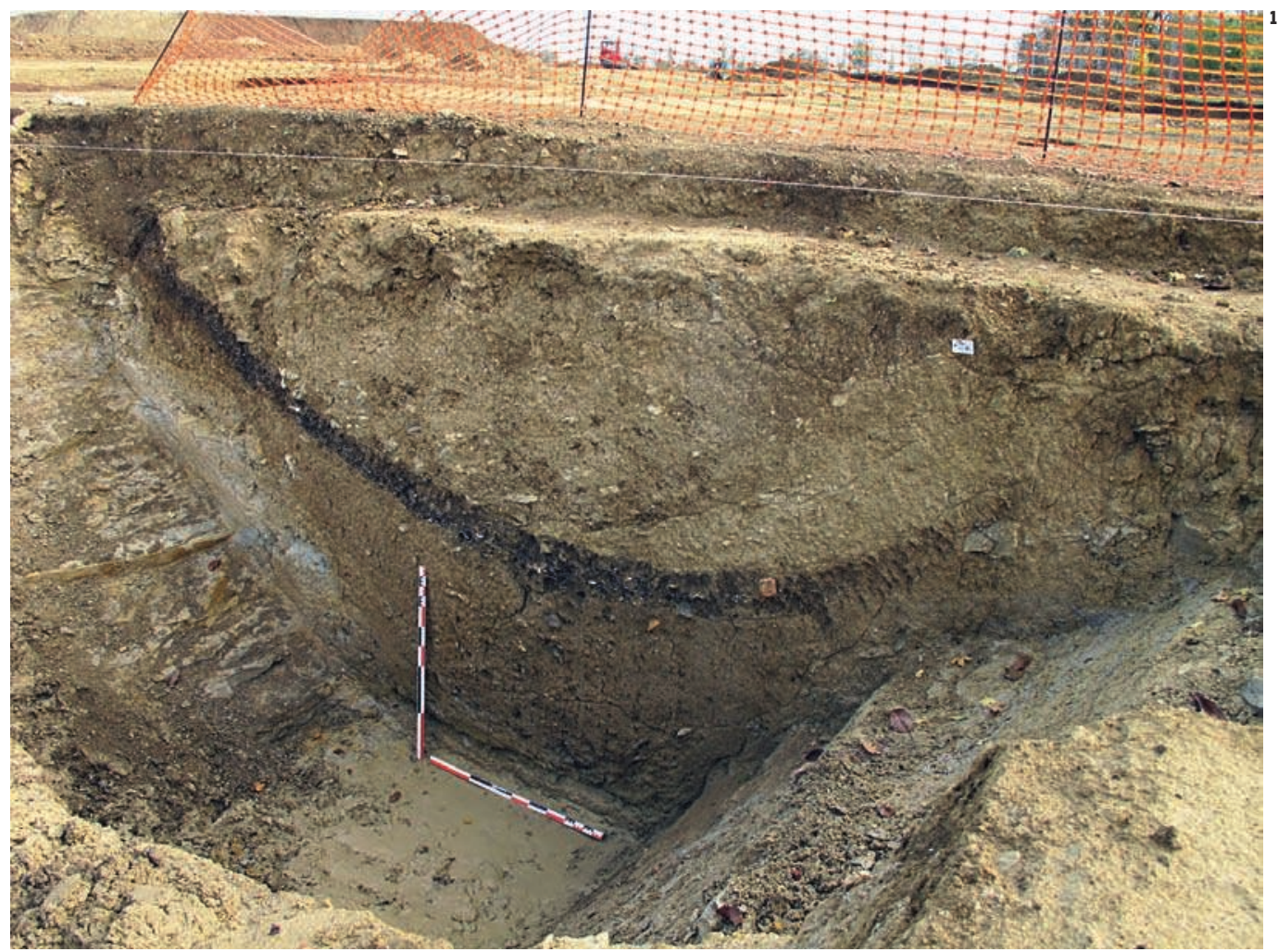




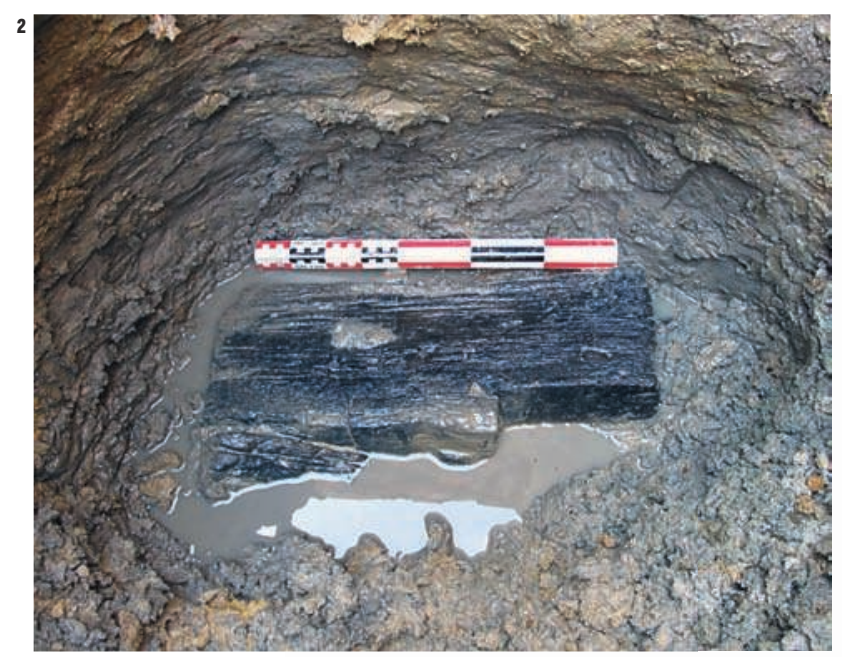

\section{Pièces de bois au fond du puits. \\ 3. Fragment de statue en calcaire avec bracelet au coude. \\ 4. Statère en or allié attribue aux Santons.}
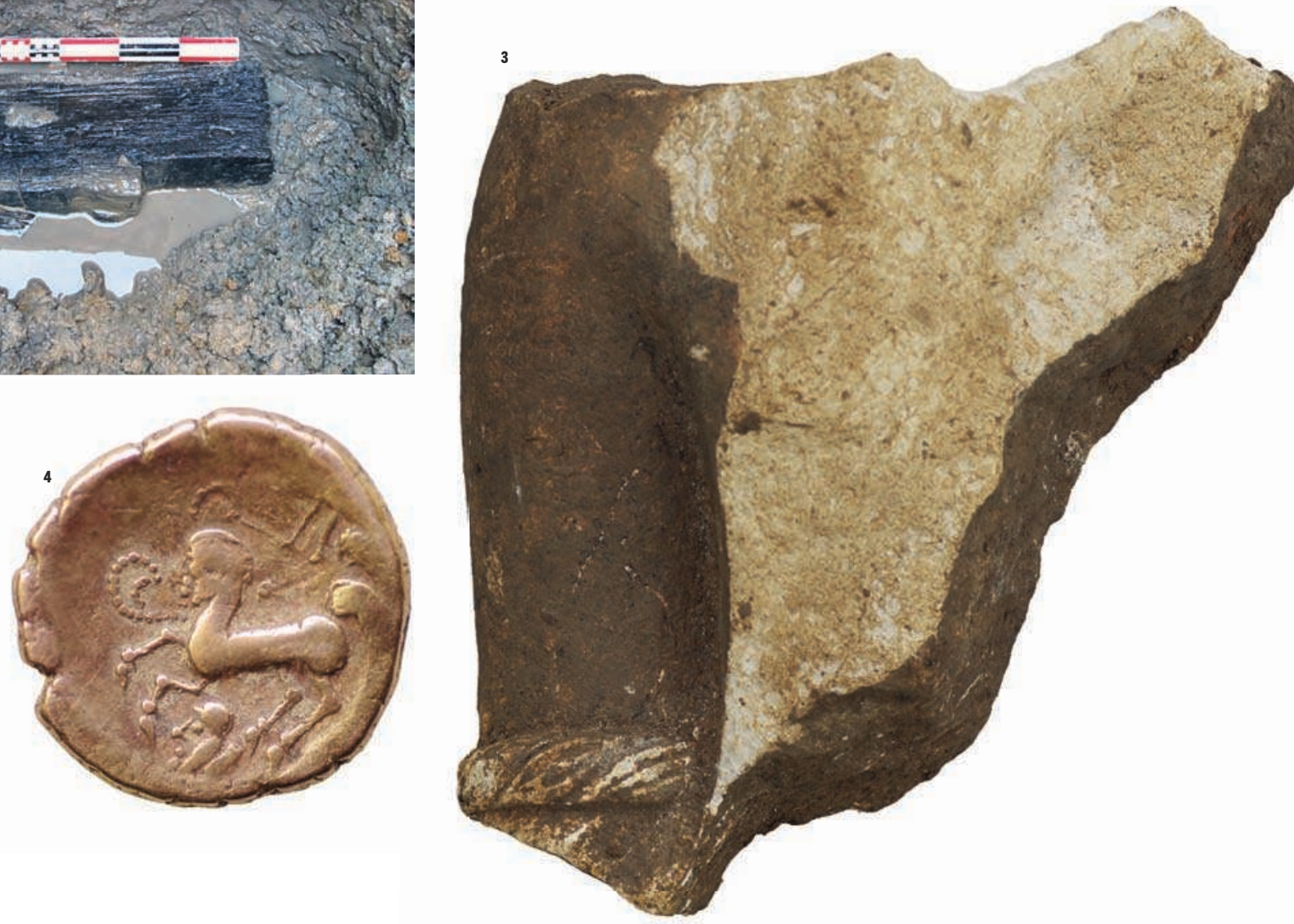

et d'abandon sous $2 \mathrm{~m}$ de remblai, très certainement pour une remise en culture de cet espace lors d'une réorganisation du territoire au début de l'époque romaine.

Le site des Grands Champs s'inscrit dans une aire géographique spécifique bien documentée en ce qui concerne les activités agricoles et littorales des pourtours des marais qui rythment la côte atlantique du centre-ouest de la Gaule (Nillesse, 2003, 2009; Landreau, 2007).

La fouille a mis en évidence

la succession de 16 séquences de construction, d'occupation ou de destruction, permettant une analyse fine de l'abondant mobilier rejeté entre le milieu du $\mathrm{III}^{\mathrm{e}}$ siècle avant notre ère et le changement d’ère. La phase la mieux documentée (fin du $\mathrm{II}^{\mathrm{e}} \mathrm{I}^{\mathrm{er}}$ siècle avant notre ère) concerne notamment le fossé d'enclos le plus imposant, avec sept séquences chronologiques successives s'étalant sur 150 ans environ. Ces séquences, qui englobent chronologiquement les événements de la guerre des Gaules, sont en revanche mal définies pour l'instant dans la région en milieu rural. Elles sont contemporaines des niveaux les plus anciens des agglomérations de Poitiers et de Saintes, qui deviendront à l'époque romaine les chefs-lieux de leur cité. Coulon se situe à la frontière de ces deux territoires, à un carrefour de communication (axe routier nord-sud et fluvial estouest). Cette position est d'autant plus stratégique qu'elle est au cœur des problématiques sur la définition et l'évolution des territoires telles qu'elles ont pu être soulevées récemment, notamment sur les zones des marais côtiers et leur spécificité (Zélie, 2003 ; Landreau, 2009 ; Landreau, à paraître). On peut s'interroger, pour finir, sur le caractère accidentel ou volontaire de l'incendie du bâtiment aristocratique. Il a lieu en tout cas à un moment où des événements politiques et militaires majeurs pour la région embrasent toute la Gaule : arrêt de la migration helvète vers la Saintonge par César en 58 avant notre ère et victoire navale romaine sur les Vénètes en 56 avant notre ère, avec l'appui de navires santons et pictons.

\section{Références bibliographiques}

LANDREAU G., 2007, « Du godet à l'auget: les établissements salicoles gaulois entre Loire et Gironde », in Bertrand I., Maguer P. (Dir.), De pierre et de Terre. Les Gaulois entre Loire et Dordogne, Catalogue de l'exposition du XXXI ${ }^{\mathrm{e}}$ colloque de l'AFEAF, Mémoire XXX, Chauvigny, Association des publications chauvinoises, p. 39-42.

LANDREAU G., ZÉlie B. (COllab.) Bardot X. (COllab.), Houdusse B. (collab.), Maratier B. (collab.), Hess S. (Collab.), Rousseau J. (COLLAB.), 2009, « Entre Isthme gaulois et Océan, la Saintonge au second âge du Fer : état des connaissances », in BERTRAND I, Duval A., Gomez de Soto J., MAguer P., Les Gaulois entre Loire et Dordogne, Actes du XXXI colloque de l'AFEAF, Chauvigny, 17-20 mai 2007, Mémoire XXXIV, t. I, Chauvigny, Association des publications chauvinoises, p. 245-306.
LANDREAU G., à paraître, Coup de pouce au pot à $\mathrm{DIOH}$, un marqueur emblématique du faciès bas-poitevin aux II $I^{e}-I^{e r}$ S. av. J.-C., Actes du congrès de la SFECAG, Poitiers, 18-20 mai 2012 Nillesse O., 2003, « Les établissements ruraux gaulois de la plaine de Luçon (Vendée) », in Plouin S., JUd P. (DIR.), Habitats, mobiliers et groupes régionaux à l'âge du Fer, Actes du XX $X^{e}$ colloque de l'AFEAF, Colmar-Mittelwihr, 16-19 mai 1996, Revue Archéologique de l'Est, Suppl. 20, p. 275-288. 